\title{
Comorbidities in rheumatic arthritis
}

\author{
Łukasz Kłodziński, Małgorzata Wisłowska
}

Clinical Department of Internal Diseases and Rheumatology, Central Clinical Hospital of the Ministry of Interior, Warsaw, Poland

\begin{abstract}
Objectives: Rheumatoid arthritis (RA) is one of the most common systemic inflammatory diseases, but its etiology is still not fully known. The aim of this preliminary study was to assess what particular comorbidities are involved in the progression of RA and determine the influence that the aforementioned diseases have on each other.

Material and methods: Forty patients with diagnosed RA according to EULAR/ACR criteria from 2010 were included in the study. The majority of the group was female $(n=35 ; 87.5 \%)$. Patients were tested using routine laboratory and imaging methods allowing diagnosis and assessment of disease activity. Dual energy X-ray absorptiometry was also evaluated for mineral density. The activity of the disease was assessed using the disease activity score DAS28 (ESR) and SDAI (Simplified Disease Activity Index).

Results: Among studied patients, based on the DAS28 index, 9 patients were in the remission phase (22.5\%) and 12 (30\%) had high disease activity. Increased values of CRP were observed in the majority of patients (65\%). The group analysis demonstrated the most common comorbidities in patients with RA, as follows: hypertension ( $n=14 ; 35 \%)$ and osteoporosis or osteopenia ( $n=13 ; 32.6 \%)$. Conclusions: Patients with rheumatoid arthritis (RA) are more susceptible to developing hypertension and osteoporosis. We did not observe a significant association between other comorbidities and activity of RA. The next study will assess a larger number of patients.
\end{abstract}

Key words: rheumatoid arthritis, comorbidities, hypertension, osteoporosis.

\section{Introduction}

Rheumatoid arthritis (RA) is an autoimmune inflammatory disease that affects up to $1.5 \%$ of the worldwide population, women in particular. RA patients suffer from progressive joint inflammation, degeneration of joints and disability as a consequence of the above-mentioned changes [1]. Because emphasis is placed on RA treatment, management of other comorbidities is usually neglected in this group of patients [2-4].

The most common comorbidities reported in the literature include cardiovascular disorders [5, 6], gastrointestinal, renal and pulmonary diseases, infections [7], osteoporosis [8], tumors and depression.

There is a correlation between the occurrence of other diseases and activity, duration and treatment of RA (especially with glucocorticosteroids - GCs) [9]. It has also been observed that certain diseases have a strong influence on the course of RA, e.g. obesity. Comorbidities are more often perceived as an adverse result of a chronic inflammatory process in RA rather than as an element affecting the course of RA. The aim of this study was to assess what particular comorbidities are involved in the progression of RA and the influence that the aforementioned diseases may have on each other.

\section{Material and methods}

Forty patients with diagnosed RA according to EULAR/ ACR criteria from 2010, recruited from the Clinical Department of Internal Medicine and Rheumatology of the Central Clinical Hospital of the Ministry of Interior in Warsaw, were included in the study. The clinical condition of the patients was evaluated on the basis of medical records and routine laboratory blood tests: white and red blood cell counts (WBC, RBC), erythrocyte sedimen- 
tation rate (ESR), C-reactive protein (CRP), total calcium concentration, rheumatoid factor, anti-citrullinated protein antibodies (ACPA), glucose, creatinine, and uric acid serum concentration. All laboratory tests were performed routinely at admission to the clinic.

Bone mineral density (BDM) was assessed by DEXA (dual-energy X-ray absorptiometry) and patients with osteoporosis and osteopenia were identified.

During the study, the activity of the disease was assessed using the disease activity score DAS28 (ESR) and SDAI (Simplified Disease Activity Index). The evaluation of the radiographic changes was based on Larsen's assessment according to the modification by Larsen-Dale, which assesses both erosions and joint space narrowing as a single score, on a scale of 0 to 5 according to reference radiographs. All data were presented as descriptive statistics (mean \pm standard deviation and percentages). The SAS 9.4 statistical software was used to compare the variables. A $p$-value $<0.05$ was considered to be statistically significant.

All participants gave informed, written consent, and the study was approved by the bioethical commission.

\section{Results}

The mean age in the group was $55.5 \pm 9.5$ years (from 31 to 68 years). $87.5 \%$ of subjects were female $(n=35)$. The clinical characteristics of patients are shown in Table I. Based on the DAS28 index, 9 patients were in remission (22.5\%) and 12 (30\%) had high disease activity (DAS28 score > 5.1). Increased values of CRP were observed in the majority of patients (65\%).

In the studied group we found that the most common comorbidities were hypertension $(\mathrm{HT})(n=14 ; 35 \%)$ and osteoporosis or osteopenia $(n=13 ; 32.6 \%)$.

From all studied patients ( $N=40), 16(40 \%)$ patients were also treated with GCS. Between patients treated with GCs, 5 (which is $31.25 \%$ of the group treated with GCs) had osteoporosis.

Amongst studied RA patients gastrointestinal diseases ( $n=9 ; 22.5 \%$ ) such as cholelithiasis, gastritis, peptic ulcer disease, diverticular disease, and hemorrhoidal disease were reported less frequently. Lung diseases occurred in $17.5 \%$ of patients; among them were diseases as interstitial lung disease, asthma, pulmonary fibrosis and infection, e.g. tuberculosis. Cardiovascular dis eases were diagnosed in $12.5 \%$ of studied patients (atrial fibrillation, sinus tachycardia with right bundle branch block, infective endocarditis or myocardial infarction). Four patients were diagnosed with cancer (10\%) and 2 with depression. Table II summarizes the incidence of comorbidities in the examined group of patients.

It was found that patients with diagnosed HT were characterized by a higher degree of disease activity assessed by DAS28 ( $p=0.0036)$ and presented higher ESR ( $p=0.0223)$ than those without HT (Table III). In addition, the mean glucose $(p=0.0242)$ and uric acid $(p=0.0019)$ serum concentration were significantly higher in these patients. Patients with osteoporosis or osteopenia confirmed by DEXA were older $(p=0.028)$, with longer duration of the disease $(p=0.0017)$ and had a lower RBC level $(p=0.0256)$ compared to patients without osteoporosis Table IV.

Table I. Clinical characteristics of rheumatoid arthritis population

\begin{tabular}{|lc|}
\hline Variable & Results \\
\hline Age, years & $55.5 \pm 9.5$ \\
\hline Female $n(\%)$ & $35(87.5)$ \\
\hline BMI & $26.6 \pm 4.7$ \\
\hline Disease duration, years & $12.5(6-17.5)$ \\
\hline DAS28 (\%) & $3.91 \pm 1.7$ \\
\hline$\leq 2.6$ & $9(22.5)$ \\
\hline$>2.6$ and $\leq 3.2$ & $2(5)$ \\
\hline$>3.2$ and $\leq 5.1$ & $17(42.5)$ \\
\hline$>5.1$ & $12(30)$ \\
\hline SDAl & $15.8 \pm 11.4$ \\
\hline Larsen-Dale scale $(\%)$ & \\
\hline 1 & $10(25)$ \\
\hline 2 & $12(30)$ \\
\hline 3 & $12(30)$ \\
\hline 4 & $6(15.0)$ \\
\hline 5 & $0(0)$ \\
\hline ESR $(\mathrm{mm} / \mathrm{h})$ & $20.5(10-39)$ \\
\hline CRP $(\mathrm{mg} / \mathrm{l})$ & $9.3(2.6-16.7)$ \\
\hline CRP $>5(\mathrm{mg} / \mathrm{l})$ & $26(65)$ \\
\hline RF $(\mathrm{IU} / \mathrm{ml})$ & $65.6(16-212)$ \\
\hline Creatinine $(\mathrm{mg} / \mathrm{dl})$ & $0.74 \pm 0.21$ \\
\hline GFR & $85.1 \pm 20.5$ \\
\hline
\end{tabular}

Table II. Incidence of comorbidities in the examined group of patients

\begin{tabular}{|lc|}
\hline Comorbidities & $n(\%)$ \\
\hline Hypertension & $14(35)$ \\
\hline Osteoporosis/osteopenia & $13(32.6)$ \\
\hline Gastrointestinal disease & $9(22.5)$ \\
\hline Lung diseases & $7(17.5)$ \\
\hline Cardiovascular diseases & $5(12.5)$ \\
\hline Malignancies & $4(10)$ \\
\hline Depression & $2(5)$ \\
\hline
\end{tabular}


Table III. Group of patients with/without hypertension and with/without osteoporosis/osteopenia

\begin{tabular}{|lccc|}
\hline Parameters & Hypertension - & Hypertension + & $p$-value \\
\hline Age & $54.3 \pm 9.8$ & $57.6 \pm 9.8$ & 0.3117 \\
\hline BMI & $25.7 \pm 3.5$ & $28.2 \pm 6.1$ & 0.0974 \\
\hline Duration of RA/years & $13(9-15)$ & $7(4-24)$ & 0.3338 \\
\hline DAS28 & $3.43 \pm 1.77$ & $4.81 \pm 1.01$ & 0.0036 \\
\hline SDAl & $13.7 \pm 12.4$ & $19.6 \pm 9.4$ & 0.1244 \\
\hline WBC $\left(\times 10^{9} / \mathrm{l}\right)$ & $7.6 \pm 2.6$ & $9.4 \pm 3.2$ & 0.0515 \\
\hline ESR $(\mathrm{mm} / \mathrm{h})$ & $16.5(9-37)$ & $36(21-56)$ & 0.0223 \\
\hline CRP $(\mathrm{mg} / \mathrm{l})$ & $8.35(2.16-14.4)$ & $10.9(5.3-30.8)$ & 0.1603 \\
\hline RF $(\mathrm{IU} / \mathrm{ml})$ & $48.8(11.4-172)$ & $118(29.1-414.1)$ & 0.0957 \\
\hline Calcium total $(\mathrm{mmol} / \mathrm{l})$ & $2.35(2.31-2.37)$ & $2.35(2.29-2.43)$ & 0.6398 \\
\hline Glucose $(\mathrm{mg} / \mathrm{dl})$ & $90.5 \pm 13.8$ & $112.7 \pm 31.8$ & 0.0242 \\
\hline Uric acid $(\mathrm{mg} / \mathrm{dl})$ & $3.84 \pm 1.17$ & $5.14 \pm 1.11$ & 0.0019 \\
\hline SBP $(\mathrm{mm} \mathrm{Hg})$ & $127 \pm 9.3$ & $135 \pm 13.7$ & 0.0284 \\
\hline DBP $(\mathrm{mm} \mathrm{Hg})$ & $76 \pm 9.7$ & $80.7 \pm 9.5$ & 0.1523 \\
\hline
\end{tabular}

$B M I$ - body mass index; SBP - systolic blood pressure; DBP - diastolic blood pressure

Table IV. Differences between patients with or without confirmed osteoporosis

\begin{tabular}{|lccc|}
\hline Parameters & Osteoporosis/osteopenia - & Osteoporosis/osteopenia + & $p$-value \\
\hline Age & $52.5 \pm 9.6$ & $61.7 \pm 5.8$ & 0.0028 \\
\hline BMI & $27.4 \pm 5.1$ & $25.1 \pm 3.2$ & 0.1579 \\
\hline Duration of RA/years & $9(5-13)$ & $18(13-25)$ & 0.0017 \\
\hline DAS28 & $3.43 \pm 1.77$ & $4.81 \pm 1.01$ & 0.9425 \\
\hline SDAl & $13.7 \pm 12.4$ & $19.6 \pm 9.4$ & 0.3909 \\
\hline RBC $\left(\times 10^{12} / \mathrm{l}\right)$ & $4.48 \pm 0.37$ & $4.2 \pm 0.32$ & 0.0256 \\
\hline ESR $(\mathrm{mm} / \mathrm{h})$ & $21(7-44)$ & $20(16-36)$ & 0.8737 \\
\hline CRP $(\mathrm{mg} / \mathrm{l})$ & $9.1(1.56-17.3)$ & $10.4(4.5-16.1)$ & 0.4793 \\
\hline RF $(I \mathrm{U} / \mathrm{ml})$ & $72(18.4-259)$ & $17(10-172)$ & 0.0920 \\
\hline Calcium total $(\mathrm{mmol} / \mathrm{l})$ & $2.35(2.32-2.42)$ & $2.34(2.29-2.36)$ & 0.6398 \\
\hline Glucose $(\mathrm{mg} / \mathrm{dl})$ & $100.9 \pm 26.4$ & $92.9 \pm 17.6$ & 0.3332 \\
\hline Uric acid $(\mathrm{mg} / \mathrm{dl})$ & $4.69 \pm 1.36$ & $3.59 \pm 0.81$ & 0.0113 \\
\hline
\end{tabular}

\section{Discussion}

Current management of RA may result in a decrease in disease activity and improvement of overall function of the patient. Nonetheless, comorbidities such as cardiovascular, kidney, lung and gastrointestinal diseases, infections, malignancies, osteoporosis and depression remain an important issue. These comorbidities are more frequently observed in patients with RA compared to the general population [9]. This is usually explained by either activity of the disease and the metabolic changes that follow or by its treatment - especially with GCs. Secondly, patients with RA do not receive sufficient medical prevention. The reason for that may be either focusing mostly on the underlying disease or paying too little attention to accompanying clinical problems. It is well illustrated by the fact that screening for breast cancer in women with RA is less frequently performed than in the overall population [2,3].

Cardiovascular diseases (CVDs) are some of the most relevant causes of death among patients with RA. Most of these patients have signs of CVD such as myocardial, pericardial or valvular diseases on autopsy. The ongoing process of inflammation predisposes to early atherosclerosis. Acute cardiovascular incidents cause a higher mortality rate among patients suffering from RA. It has been shown that traditional risk factors such as high 
BMI, diabetes mellitus (DM), increased blood pressure and smoking, as well as nontraditional ones such as RA disease duration, activity and seropositivity, increase the risk of CVD among RA patients. In this group, however, decreased lipid concentrations are associated with an increased risk of occurrence of CV events as opposed to the overall population [10]. Insulin resistance also seems to play a role among our group of patients since it has been shown to be an independent risk factor for developing CVD. The incidence of DM in patients with RA, however, is not increased. Another independent risk factor for CVD is smoking, which is also associated with an increase in activity of RA [10]. Aviña-Zubieta et al. [11] searched the databases Medline, Embase, LILACS and Cochrane to determine the risk of incident CVD compared to the general population and analyzed observational studies that included 41,490 patients. The authors showed that there is a $48 \%$ increased risk of CVD in RA patients compared to the general population. In our research we found that $35 \%$ of patients have HT and $12.5 \%$ of them have other CVDs.

In the studied population there were several patients with $\mathrm{HT}$, which is known to be a modifiable cardiovascular risk factor [12]. It has been shown that HT prevalence in the overall RA patient population is comparable or higher than in the population of elderly patients (above 65 years of age) in England [13]. What is more, metabolic changes that occur in the course of RA (especially inflammation), their consequences (physical inactivity) and drugs administered to patients as treatment seem to have an influence on the development and progression of HT $[13,14]$.

Drugs commonly used in patients with CVD - statins - have an influence on the course of RA, reducing the inflammatory marker levels (e.g. CRP). Moreover, this group of drugs have both anti-inflammatory and immunomodulatory effects that may affect the inflammatory process in rheumatoid disorders. It seems crucial to diagnose CVDs and manage them properly as soon as possible among patients with RA as CVD risk is higher in early stages of RA. It has been shown that the CVD risk increases with the activity and duration of the underlying disease [10].

Due to their powerful anti-inflammatory and pain relief effects, non-steroid anti-inflammatory drugs (NSAIDs) are frequently included in the treatment of RA patients. They should be, however, given carefully to patients with a history of prior myocardial events as there is a risk of recurrence of the cardiovascular event. Steroids, on the other hand, in spite of their important role in the treatment of RA, influence the traditional CVD risk factors in a dose-dependent manner.

Another important comorbidity that occurs among RA patients is depression. Its prevalence in the group of patients whose physical health is handicapped is twoto threefold higher than in the population of physically healthy people. A combination of a psychiatric disorder and rheumatoid disease may cause severe problems and worsen the symptoms of both conditions [15]. Current views and research results show the relationship between the development of depression and inflammatory activity and the production of a number of proinflammatory cytokines, such as IL-17, IL-6, and TNF [16]. As well as the development of a chronic disease with pain and disability, it has an impact on the behavior and development of symptoms of depression

Almost one third of the patients in the studied population suffered from osteoporosis and osteopenia. In the group of RA patients bone mineral density (BMD) loss may increase in the early stage of the disease, regardless of use of GCs, and it is associated with the levels of inflammatory markers [17], among them RANK-L, a strong osteoclast-stimulating factor. In the study by Bilgici et al. [18] on 304 of patients with RA, osteoporosis was present in 91 patients (29.9\%). In our research we obtained comparable results: $30.7 \%$ of patients with osteoporosis, and $69.3 \%$ with osteopenia.

Glucocorticosteroids are used commonly in treatment of RA. In medium and high doses they significantly increase the risk of developing osteoporosis. It seems obvious that asymptomatic vertebral fractures increase with age, but also the adjusted prevalence in RA was quite high, 33.78\% for rheumatoid arthritis in the study by Angeli et al. [19]. What was interesting, the authors did not find a significant association with GCs cumulative dose and duration of therapy. However, as shown in other studies, GC-induced osteoporosis (GIOP) may occur with asymptomatic fractures [20]. Prevention of GIOP using active calcium, vitamin D and bisphosphonates is indicated.

As for internal diseases that occur as comorbidities in RA patients, gastric and duodenal ulcers seem to be a prevalent complication. In the present group $22 \%$ of patients had some diseases affecting the gastrointestinal tract as mentioned above: cholelithiasis, gastritis, peptic ulcer disease, diverticular disease, hemorrhoidal disease. However, they cannot be clearly associated only with the occurrence of RA, as age, NSAID treatment or other risk factors may have influenced their development. It is undoubtedly related to the use of NSAIDs in the treatment of pain and rheumatoid diseases [21]. Moreover, the use of biological drugs such as tocilizumab and tofacitinib is associated with increased incidence of gastrointestinal perforation, which was not shown in our study.

Kidney function in patients with RA also deteriorates over time, which may be linked with CVDs - a comorbidity that has already been mentioned. On the other hand, abnormalities in kidney physiology may lead to 
an increase in morbidity from CVDs [22]. Other internal diseases that may develop in the course of RA include intestinal lung diseases [23], whose occurrence correlates with increased morbidity and mortality in RA. In the study group, $17.5 \%$ of patients had confirmed lung involvement, which prevails in comparison with cardiovascular symptoms indicated in $12.5 \%$ of subjects. In the Bilgici et al. [18] study of 52 patients with RA as many as $40 \%$ of the patients had symptoms of lung disease.

We did not find any correlation between the activity or duration of RA and the incidence of malignancies in our group of patients. Yet there are some studies showing such links in larger populations. There have been speculations that drugs used in rheumatoid diseases' treatment (especially GCs and biologic drugs) may increase the longterm risk of developing a tumor due to their suppressive effect on the immune system [24]. Indeed, it seems that patients with RA are more prone to developing lung and lymphoma malignancies than the overall population [25]. It has also been shown that treatment with certain groups of drugs (e.g. cyclophosphamide, methotrexate) results in an increased risk of developing specific malignancies [26, 27]. Undoubtedly, screening for malignancies should be an important part of follow-up in the case of patients treated with immunosuppressive drugs. Since drugs affecting the immune system, as well as synthetic and biological agents, are used in the treatment of RA, it is important to take into consideration the potential risk of infections in this group of patients [28]. The symptoms and consequences of the underlying disease, such as restricted mobility, joint damage and skin lesions, may result in a higher risk of developing infections in this group of patients. Moreover, GCs have been proved to increase the prevalence of infections in RA patients. The same effect has also been shown for certain risk factors such as age $\geq 70$, class III or IV of RA, DAS28-ESR $\geq 3.8$ and use of biological drugs, the last three factors being related to the disease activity [29]. In the present group no active infections were found. Further studies on bigger groups of patients are required.

\section{Conclusions}

To sum up our preliminary study, patients with rheumatoid arthritis are primarily susceptible to developing $\mathrm{HT}$, osteoporosis and osteopenia. Therefore we believe that screening for detection of the selected comorbidities should be a part of the standard diagnostic procedure for patients with rheumatic arthritis. In clinical practice there should be more specific identification of patients with risk factors for comorbidities.

The authors declare no conflict of interest.

\section{References}

1. Khurana R, Berney SM. Clinical aspects of rheumatoid arthritis. Pathophysiology 2005; 12: 153-165.

2. Dougados M, Soubrier M, Antunez A, et al. Prevalence of comorbidities in rheumatoid arthritis and evaluation of their monitoring: results of an international, cross-sectional study (COMORA). Ann Rheum Dis 2014; 73: 62-68.

3. MacLean $\mathrm{CH}$, Louie $\mathrm{R}$, Leake $\mathrm{B}$, et al. Quality of care for patients with rheumatoid arthritis. JAMA 2000; 284: 984-992.

4. Curtis JR, Arora T, Narongroeknawin P, et al. The delivery of evidence-based preventive care for older Americans with arthritis. Arthritis Res Ther 2010; 12: R144.

5. Peters MJ, Symmons DP, McCarey D, et al. EULAR evidence-based recommendations for cardiovascular risk management in patients with rheumatoid arthritis and other forms of inflammatory arthritis. Ann Rheum Dis 2010; 69: 325-331.

6. Nurmohamed MT, Heslinga M, Kitas GD. Cardiovascular comorbidity in rheumatic diseases. Nat Rev Rheumatol 2015; 11 : 693-704.

7. van Assen S, Agmon-Levin N, Elkayam O, et al. EULAR recommendations for vaccination in adult patients with autoimmune inflammatory rheumatic diseases. Ann Rheum Dis 2011; 70: 414-422.

8. Kanis JA, Burlet N, Cooper C, et al. European guidance for the diagnosis and management of osteoporosis in postmenopausal women. Osteoporos Int 2008; 19: 399-428.

9. Baillet A, Gossec L, Carmona L, et al. Points to consider for reporting, screening for and preventing selected comorbidities in chronic inflammatory rheumatic diseases in daily practice: a EULAR initiative. Ann Rheum Dis 2016; 75: 965-973.

10. Sen D, González-Mayda M, Brasington RD Jr. Cardiovascular disease in rheumatoid arthritis. Rheum Dis Clin North Am 2014; 40: 27-49.

11. Aviña-Zubieta, Abrahamowicz M, De Vera MA, et al. Immediate and past cumulative effects of oral glucocorticoids on the risk of acute myocardial infarction in rheumatoid arthritis: a population-based study. Rheumatology (Oxford) 2013; 52: 68-75.

12. Yusuf S, Hawken S, Ounpuu S, et al. Effect of potentially modifiable risk factors associated with myocardial infarction in 52 countries (the INTERHEART study): case-control study. Lancet 2004; 364: 937-952.

13. Panoulas VF, Metsios GS, Pace AV, et al. Hypertension in rheumatoid arthritis. Rheumatology (Oxford) 2008; 47: 1286-1298.

14. Metsios GS, Stavropoulos-Kalinoglou A, Veldhuijzen van Zanten JJ, et al. Rheumatoid arthritis,cardiovascular disease and physical exercise: a systematic review. Rheumatology (Oxford) 2008; 47: 239-248.

15. National Collaborating Centre for Mental Health (UK). Depression in Adults with a Chronic Physical Health Problem: Treatment and Management. Leicester (UK): British Psychological Society 2010.

16. Young JJ, Bruno D, Pomara N. A review of the relationship between proinflammatory cytokines and major depressive disorder. J Affect Disord 2014; 169: 15-20.

17. Duru N, van der Goes MC, Jacobs JW, et al. EULAR evidence-based and consensus-based recommendations on the 
management of medium to high-dose glucocorticoid therapy in rheumatic diseases. Ann Rheum Dis 2013; 72: 1905-1913.

18. Bilgici A, Ulusoy $\mathrm{H}$, Kuru $\mathrm{O}$, et al. Pulmonary involvement in rheumatoid arthritis. Rheumatol Int 2005; 25: 429-435.

19. Angeli A, Guglielmi G, Dovio A, et al. High prevalence of asymptomatic vertebral fractures in post-menopausal women receiving chronic glucocorticoid therapy: a cross-sectional outpatient study. Bone 2006; 39: 253-259.

20. Curtis JR, Westfall AO, Allison J, et al. Population-based assessment of adverse events associated with long-term glucocorticoid use. Arthritis Rheum 2006; 55: 420-426.

21. Lanza FL, Chan FK, Quigley EM; Practice Parameters Committee of the American College of Gastroenterology. Guidelines for prevention of NSAID-related ulcer complications. Am J Gastroenterol 2009; 104: 728-738.

22. Hickson LJ, Crowson CS, Gabriel SE, et al. Development of reduced kidney function in rheumatoid arthritis. Am J Kidney Dis 2014; 63: 206-213.

23. Giles JT, Danoff SK, Sokolove J, et al. Association of fine specificity and repertoire expansion of anticitrullinated peptide antibodies with rheumatoid arthritis associated interstitial lung disease. Ann Rheum Dis 2014; 73: 1487-1494.
24. Wilton KM, Matteson EL. Malignancy Incidence, Management, and Prevention in Patients with Rheumatoid Arthritis. Rheumatol Ther 2017; 4: 333-347.

25. Simon TA, Thompson A, Gandhi KK, et al. Incidence of malignancy in adult patients with rheumatoid arthritis: a meta-analysis. Arthritis Res Ther 2015; 17: 212.

26. Radis CD, Kahl LE, Baker GL, et al. Effects of cyclophosphamide on the development of malignancy and on long-term survival of patients with rheumatoid arthritis. A 20-year follow up study. Arthritis Rheum 1995; 38: 1120-1127.

27. Buchbinder R, Barber M, Heuzenroeder L, et al. Incidence of melanoma and other malignancies among rheumatoid arthritis patients treated with methotrexate. Arthritis Rheum 2008; 59: 794-799.

28. Van Albada-Kuipers GA, Linthorst J, Peeters EAJ, et al. Frequency of infection among patients with rheumatoid arthritis versus patients with osteoarthritis or soft tissue rheumatism. Arthritis Rheum 1988; 31: 667-671.

29. Hashimoto A, Suto S, Horie K, et al. Incidence and Risk Factors for Infections Requiring Hospitalization, Including Pneumocystis Pneumonia, in Japanese Patients with Rheumatoid Arthritis. Int J Rheum 2017; 2017: 6730812. 